\title{
Hypomagnetic field, an ignorable environmental factor in space?
}

\author{
MO WeiChuan, LIU Ying* \& HE RongQiao \\ State Key Laboratory of Brain and Cognitive Sciences, Institute of Biophysics, Chinese Academy of Sciences, Beijing 100101, China
}

Received April 19, 2014; accepted April 24, 2014; published online May 12, 2014

Citation: Mo WC, Liu Y, He RQ. Hypomagnetic field, an ignorable environmental factor in space?. Sci China Life Sci, 2014, 57: 726-728, doi: 10.1007/s11427-014-4662-x

All living organisms on the earth have been exposed to the geomagnetic field (GMF, $\sim 50 \mu \mathrm{T})$, a nature component of the habitability environment, throughout the evolutionary history. However, the environmental magnetic field (MF) in space decreases geometrically with the distance from the ground. The MF magnitude $(|\mathrm{B}|)$ at the International Space Station (ISS; near-earth orbit) is similar to the GMF ( $80 \%$ GMF with limited variation), except that the radial component $\left(B_{r}\right)$ exhibits significant variations both in direction and magnitude [1]. The environmental MF in outer space is extremely lower than the GMF, called a hypomagnetic field (HMF), e.g., Martian MF is $<5 \mu \mathrm{T}$, Lunar MF is $<300 \mathrm{nT}$ and interplanetary MF is just a few nanotesla [2] (Figure 1). Thus, besides micro-gravity ( $\mu$-gravity) and cosmic radiation, astronauts have to be exposed and adapt to the HMF condition when onboard in the long-term and long-distance space missions.

Even though biological effects of the HMF were valued in early studies (1960s), exploration of the space HMF has not rapidly progressed in the following decades. The reasons may be as follows. First, considering the strength of MF in the near-earth orbit is about $80 \%$ of the GMF, the effects of $\mu$-gravity and radiation are more observable than HMF in the short-term and short-distance space missions. Significant attention has been attached to the ground-based simulation experiments of $\mu$-gravity and dosimetry of radiation in the previous space projects and missions. Second, the maintenance of a high quality HMF requires sophisticated instrument and expensive materials, such as highprecision magnetometer, magnetic shielding metal and

*Corresponding author (email: liuy@ moon.ibp.ac.cn)

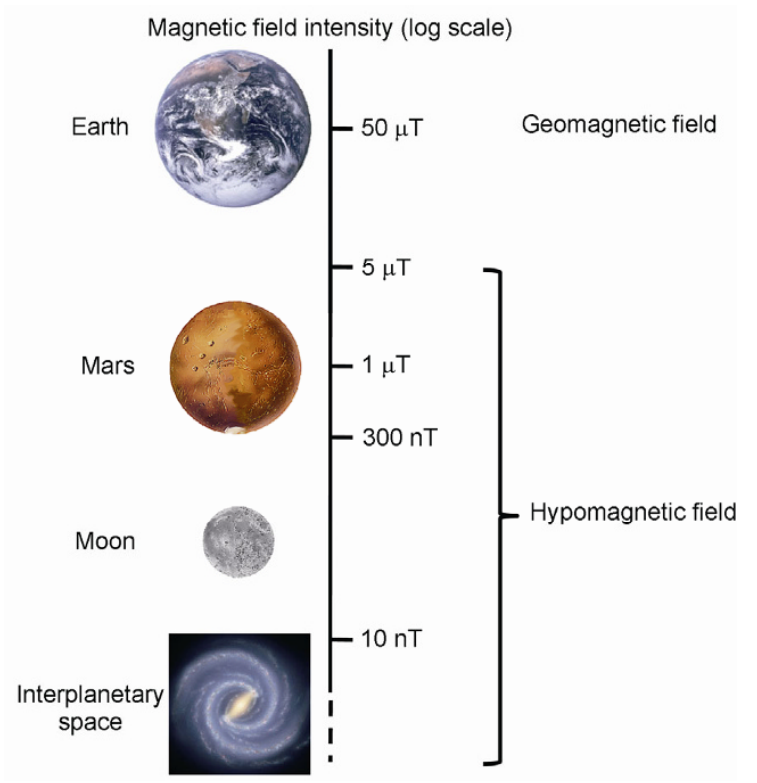

Figure 1 The environmental magnetic field in outer space and the definition of the hypomagnetic field. A weak static magnetic field with $|\mathrm{B}|<5 \mu \mathrm{T}$ is defined as a hypomagnetic field (HMF). Lunar magnetic field range: $<300 \mathrm{nT}$; Martian magnetic field range: $300 \mathrm{nT}-5 \mu \mathrm{T}$. The interplanetary magnetic field is lower than $10 \mathrm{nT}$. The magnetic intensity is plotted in logarithmic scale (modified from the figure in [2]).

Helmholtz coils system. In the previous studies, the varied conditions of the applied experimental set ups often raise additional concerns in the reproducibility and comparability [2]. Third, effects of HMF are weak or reversible in many cases although some biological responses are remarkably found in recent years. It has been proposed that gravity may 
conceal the HMF effects in the ground-based HMF simulation experiments. Unfortunately, the reports on the ISS-based HMF trials are very limited to date. The overlooked HMF effects might indeed bring great risk to the health of astronauts during the long-term journey in outer space.

So far, with the development of advanced and standardized HMF experimental systems, interesting studies on the effects of the HMF have consequently arisen. Significant findings on the effects of the HMF have been reported in the recent decade. A number of experiments have made it obvious that the HMF greatly disturbs the functional state of organisms [2,3]. It has been shown that HMF exposure alters the embryonic development, cell proliferation, oxidative stress state, and the function of immune system [2,3]. The HMF-exposed animals/human subjects exhibited impaired learning and memory, reduced cognitive ability and working capacity. Recently, we performed a transcriptome profiling assay on the HMF-exposed human neuroblastoma cells and found that HMF exposure significantly affects the transcription of genes related to macromolecule transport, metabolic process and mRNA processing, and to the subsequent pathways involved in the organization of the cytoskeleton, regulation of chromatin condensation, transcription, and brain function [4]. Given the reported adverse impacts of the HMF on many aspects of the life system, espe- cially the brain functions, astronauts are exposed to the $\mathrm{HMF}$ and thus to potential health risks during interplanetary navigation. Thus, it is necessary to elucidate the mechanism of the HMF effect and to develop effective strategies to counteract the HMF for the life support and health care of the astronauts in outer space.

Actually, the space $\mu$-gravity, radiation and HMF act on multiple aspects of an organism including bone metastasis, brain and cognition, immune system, cell growth and embryonic development, cytoskeleton, stability of genetic materials (e.g., epigenetic changes), and oxidative stress responses (Table S1 in Supporting Information), which implicates the presence of interactions among the three factors. In the current experiments, the combined effects of radiation with $\mu$-gravity have been considered, while the interaction of HMF with radiation or $\mu$-gravity on an organism has not attracted enough attention. However, in the studies on the ground-based instrumentation, the effects of $\mu$-gravity and HMF are in fact often mixed with GMF and normal gravity, respectively. For example, the potential effect of GMF is largely overlooked in the observations of the $\mu$-gravity effects with common $\mu$-gravity simulation approaches (e.g., a 3D-clinostat). Moreover, we have observed enhanced gravitropism in the germinating soybean seeds by excluding the interference from GMF under a ground-based HMF simulation system [5]. Therefore, it would also be

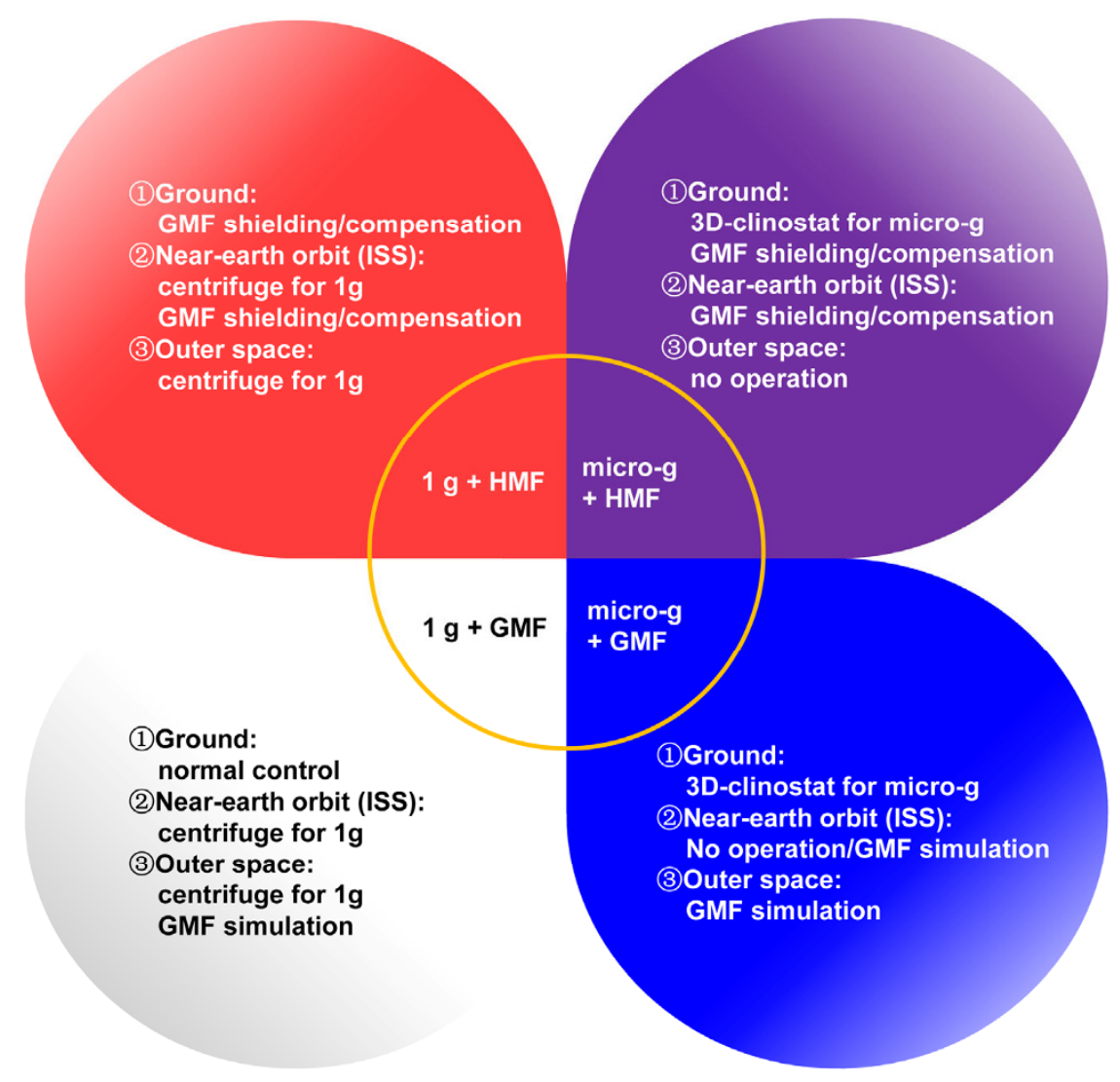

Figure 2 The experimental concept to clarify the biological effects of $\mu$-gravity and HMF under different experimental environments. 
reasonable to clarify the interference of GMF when investigating the biological effect of $\mu$-gravity at the ground-based experiments, e.g., settle a $2 \mathrm{D}$ or 3D-clinostat in a magnetic shielding chamber (a HMF environment), and vice versa (Figure 2). Thus, the magneto-gravity effect offers the necessity and possibility for the initiation of the study on the combinational effects of $\mu$-gravity, radiation and HMF in the further scientific projects, in addition to the practical need to foresee the real risk in space. The biological responses to the three factors should be carefully distinguished by using combined experimental set-ups, which expands their function beyond just simulating the "true" space environment. The design of a feasible, practical and robust experimental set-up which can precisely provide the complex combinations of ionizing radiation, $\mu$-gravity and/or HMF is critical to improving the progress in space life science. The close cooperation between expert biolo- gists and engineers will be greatly valuable and imperative.

1 Del Seppia C, Mezzasalma L, Messerotti M, Cordelli A, Ghione S. Simulation of the geomagnetic field experienced by the International Space Station in its revolution around the Earth: effects on psychophysiological responses to affective picture viewing. Neurosci Lett, 2006, 400: 197-202

2 Mo WC, Liu Y, He RQ. A biological perspective of the hypomagnetic field: from definition towards mechanism. Prog Biochem Biophys, 2012, 39: 835-842

3 Jia B, Shang P. Research progress of biological effects of hypomagnetic fields. Space Med Med Eng, 2009, 22: 308-312

4 Mo WC, Liu Y, Bartlett PF, He RQ. Transcriptome profile of human neuroblastoma cells in the hypomagnetic field. Sci China Life Sci, 2014, 57: 448-461

5 Mo WC, Zhang ZJ, Liu Y, Zhai GJ, Jiang YD, He RQ. Effects of a hypogeomagnetic field on gravitropism and germination in soybean. Adv Space Res, 2011, 47: 1616-1621

Open Access This article is distributed under the terms of the Creative Commons Attribution License which permits any use, distribution, and reproduction in any medium, provided the original author(s) and source are credited.

\section{Supporting Information}

Table S1 The biological effects of the risking factors in outer space

The supporting information is available online at life.scichina.com and link.springer.com. The supporting materials are published as submitted, without typesetting or editing. The responsibility for scientific accuracy and content remains entirely with the authors. 